\title{
The optical afterglow and host galaxy of GRB 000926
}

\author{
J. U. Fynbo ${ }^{1}$, J. Gorosabel ${ }^{2}$, T. H. Dall ${ }^{3,4}$, J. Hjorth ${ }^{5}$, H. Pedersen ${ }^{5}$, M. I. Andersen ${ }^{6}$, P. Møller ${ }^{1}$,
} S. Holland ${ }^{11}$, I. Smail ${ }^{7}$, N. Kobayashi ${ }^{8}$, E. Rol ${ }^{9}$, P. Vreeswijk ${ }^{9}$, I. Burud ${ }^{10}$, B. L. Jensen ${ }^{5}$, B. Thomsen ${ }^{4}$, A. Henden ${ }^{12}$, F. Vrba ${ }^{12}$, B. Canzian ${ }^{12}$, J. M. Castro Cerón ${ }^{13}$, A. J. Castro-Tirado ${ }^{14,15}$, T. Cline ${ }^{19}$, M. Goto ${ }^{9}$, J. Greiner ${ }^{17}$, M. T. Hanski ${ }^{18}$, K. Hurley ${ }^{16}$, N. Lund ${ }^{2}$, T. Pursimo ${ }^{18}$, R. Østensen ${ }^{20}$, J. Solheim ${ }^{20}$, N. Tanvir ${ }^{21}$, and H. Terada ${ }^{22}$

1 European Southern Observatory Karl-Schwarzschild-Straße 2, 85748 Garching, Germany

2 Danish Space Research Institute, Juliane Maries Vej 30, 2100 Copenhagen Ø, Denmark

${ }^{3}$ Nordic Optical Telescope, Apartado Postal 474, 38700 Santa Cruz de La Palma, Spain

4 Institute of Physics and Astronomy, University of Århus, 8000 Århus C, Denmark

5 Astronomical Observatory, University of Copenhagen, Juliane Maries Vej 30, 2100 Copenhagen $\varnothing$, Denmark

6 Division of Astronomy, PO Box 3000, 90014 University of Oulu, Finland

7 Department of Physics, University of Durham, South Road, Durham DH1 3LE, UK

8 SUBARU Telescope, National Astronomical Observatory of Japan, 650 North A'ohoku Place Hilo, Hawaii 96720, USA

9 University of Amsterdam, Kruislaan 403, 1098 SJ Amsterdam, The Netherlands

10 Institut d'Astrophysique et de Géophysique, Université de Liège, Avenue de Cointe 5, 4000 Liège, Belgium

11 Department of Physics, University of Notre Dame, Notre Dame, IN 46556-5670, USA

12 Universities Space Research Association U.S. Naval Observatory, Flagstaff Station, Flagstaff, AZ 86002-1149, USA

13 Real Instituto y Observatorio de la Armada, Sección de Astronomía, 11110 San Fernando-Naval, Cádiz, Spain

14 Instituto de Astrofísica de Andalucía (IAA-CSIC), PO Box 03004, 18080 Granada, Spain

15 Laboratorio de Astrofísica Espacial y Física Fundamental (LAEFF-INTA), PO Box 50727, 28080, Madrid, Spain

16 University of California, Berkeley, Space Sciences Laboratory, Berkeley, CA 94720-7450, USA

17 Astrophysical Institute Potsdam, An der Sternwarte 16, 14482 Potsdam, Germany

18 Tuorla Observatory, University of Turku, 21500 Piikkiö, Finland

19 NASA Goddard Space Flight Center, Code 661, Greenbelt, MD 20771, USA

20 Department of Physics, University of Troms $\varnothing$, Troms $\varnothing$, Norway

21 Department of Physical Sciences, University of Hertfordshire, College Lane, Hatfield, Herts AL10 9AB, UK

22 Department of Physics, Kyoto University, Kitashirakawa Oiwake-cho, Sakyo, Kyoto 606-8502, Japan

Received 9 February 2001 / Accepted 6 April 2001

\begin{abstract}
We present the discovery of the Optical Transient (OT) of the long-duration gamma-ray burst GRB 000926. The optical transient was detected independently with the Nordic Optical Telescope and at Calar Alto 22.2 hours after the burst. At this time the magnitude of the transient was $R=19.36$. The transient faded with a decay slope of about 1.7 during the first two days after which the slope increased abruptly (within a few hours) to about 2.4. The light-curve started to flatten off after about a week indicating the presence of an underlying extended object. This object was detected in a deep image obtained one month after the GRB at $R=23.87 \pm 0.15$ and consists of several compact knots within about 5 arcsec. One of the knots is spatially coincident with the position of the OT and hence most likely belongs to the host galaxy. Higher resolution imaging is needed to resolve whether all the compact knots belong to the host galaxy or to several independent objects. In a separate paper we present a discussion of the optical spectrum of the OT, and its inferred redshift (Møller et al., in prep.).
\end{abstract}

Key words. cosmology: observations - gamma rays: bursts

\footnotetext{
Send offprint requests to: J. U. Fynbo, e-mail: jfynbo@eso.org

* Based on observations made with the Nordic Optical Telescope, operated on the island of La Palma jointly by Denmark, Finland, Iceland, Norway, and Sweden.

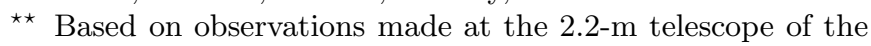
German-Spanish Calar Alto Observatory.
}

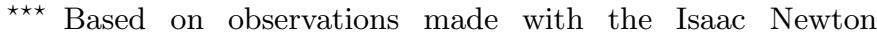
Telescope operated on the island of La Palma by the Isaac Newton Group in the Spanish Observatorio del Roque de los Muchachos of the Instituto de Astrofisica de Canarias. 


\section{Introduction}

The discoveries of the first X-ray afterglow (Costa et al. 1997) and OT (van Paradijs et al. 1997) of a gammaray burst (GRB) have led to a major breakthrough in GRB research. The determination of a redshift of 0.835 for GRB 970508 (Metzger et al. 1997), and the subsequent determination of redshifts of more than a dozen bursts with a median redshift of $\sim 1.1$, have firmly established their cosmological origin (e.g. Kulkarni et al. 2000; Castro-Tirado 2001 and references therein). One of the current goals is to use the OT properties (brightness, position within the host galaxy, light-curve shape) as diagnostic tools to study the environment of GRBs and to possibly shed some light on the nature of GRB progenitors (e.g. Bloom et al. 2000).

Another very appealing aspect of GRB research is the use of GRBs as cosmological probes. GRBs have been suggested as probes of the very high redshift universe (Lamb \& Reichart 2000; Andersen et al. 2000). An equally interesting use of GRBs is as an independent way by which to select galaxies at cosmological distances. In some widely accepted scenarios GRBs are related to deaths of very massive, short-lived stars and furthermore gamma-rays are not obscured by dust. Hence a sample of GRB host galaxies may be considered star-formation-selected independent of the amount of extinction of the rest-frame UV and optical emission. However, we do not know if GRBs (as a class) evolve with redshift and furthermore GRBs are of course gamma-ray flux selected. It is nevertheless interesting to compare GRB-selected galaxies with other samples of galaxies selected e.g. by rest-frame UV flux (Steidel et al. 1996), Damped Ly- $\alpha$ Absorption (DLAs, e.g. Wolfe et al. 1995; Djorgovski et al. 1996; Møller \& Warren 1993, 1998; Ellison et al. 2001), Ly- $\alpha$ emission (Hu et al. 1999; Fynbo et al. 2000a; Kudritzki et al. 2000; Steidel et al. 2000) or sub-mm emission (e.g. Ivison et al. 2000). There is currently some controversy as to whether most of the star-formation at high redshift takes place in the UV-selected or in the sub-mm selected objects (e.g. Smail et al. 1997; Hughes et al. 1998; Peacock et al. 2000; Adelberger \& Steidel 2000; van der Werf et al. 2000). DLAs trace a very abundant population of (proto)galaxies at high redshift that could also contribute significantly to the star-formation density (Fynbo et al. 1999, 2000a). A well understood sample of GRB host galaxies may resolve where most of the stars are formed as a function of redshift.

In this paper we present the detection of the OT and host galaxy of GRB 000926 and the results of multi-colour optical and near infrared (IR) photometry. We then compare the properties of GRB 000926 and its host galaxy with those of GRB $000301 \mathrm{C}$, which occurred at nearly the same redshift, with emphasis on the (very different) properties of their host galaxies.

Throughout this paper, we adopt a Hubble constant of $H_{0}=65 \mathrm{~km} \mathrm{~s}^{-1} \mathrm{Mpc}^{-1}$ and assume $\Omega_{\mathrm{m}}=0.3$ and $\Omega_{\Lambda}=0.7$.

\section{Observations}

GRB 000926 was detected by three instruments in the Interplanetary Network (IPN: Ulysses, Konus-WIND, and NEAR), and localized to a $35 \operatorname{arcmin}^{2}$ error box which was circulated via the GRB Coordinates Network $(\mathrm{GCN})^{1}$ 20.3 hours after the burst (Hurley 2000). The Earthcrossing time for the burst was September 26.9927 UT. As observed by Ulysses, it had a duration of approximately $25 \mathrm{~s}$ (placing it in the "long duration" burst category), a revised 25-100 keV fluence of $6.2 \times 10^{-6} \mathrm{erg} \mathrm{cm}^{-2}$, and a revised peak flux over $0.25 \mathrm{~s}$ of $8.6 \times 10^{-7} \mathrm{erg} \mathrm{cm}^{-2} \mathrm{~s}$.

The error-box of GRB 000926 (Hurley 2000) was observed in the $R$-band with the 2.56-m Nordic Optical Telescope (NOT) and the Calar Alto (CA) 2.2-m telescope on 2000 September 27.85 UT (20.64 hours after the burst). Comparing with red Palomar Optical Sky Survey II exposures an OT was found in the error box (Gorosabel et al. 2000; Dall et al. 2000). Spectra were obtained at the NOT on both September 27 and September 28; these spectra revealed a strong metal absorption system at a redshift of $z=2.0375 \pm 0.0007$, which in all likelihood is due to gas in the GRB host galaxy (Møller et al., in prep). The OT was observed during the following weeks at the NOT, CA and also with the Isaac Newton Telescope (INT), the $1.0 \mathrm{~m}$ telescope at the US Naval Observatory at Flagstaff Station (USNOFS), and in the IR with the 8.2-m Subaru telescope and the 3.8-m United Kingdom Infrared Telescope (UKIRT). The logs of optical and IR observations are given in Tables 2 and 5 . A finding-chart and $R$-band images of the OT at three different epochs are shown in Fig. 1.

Radio and X-ray afterglow measurements have been reported by Frail et al. (2000) and by Piro et al. (2000). Optical afterglow measurements have also been reported by Price et al. (2001). This paper includes preliminary reduced NOT and CA observations from Hjorth et al. (2000), Fynbo et al. (2000b) and Gorosabel et al. (2000) for which the finally reduced data appear in this paper.

\section{Celestial position}

By measuring the position of the OT relative to 80 stars in the USNO-A2.0 catalog we found the celestial coordinates of the OT to be RA(J2000) = 17:04:09.68, Dec(J2000) $=$ $+51: 47: 10.5$ with an internal error of about 0.1 arcsec and a systematic error of about 0.25 arcsec (Deutsch 1999).

\section{Photometry}

\subsection{R-band light-curve of the OT}

In comparison with GRB 000301C (Jensen et al. 2001) GRB 000926 was found to be hosted by a relatively luminous host galaxy (see Sect. 6). When measuring the magnitude of the OT directly on the images with Point

\footnotetext{
1 http://gcn.gsfc.nasa.gov/gcn/
} 

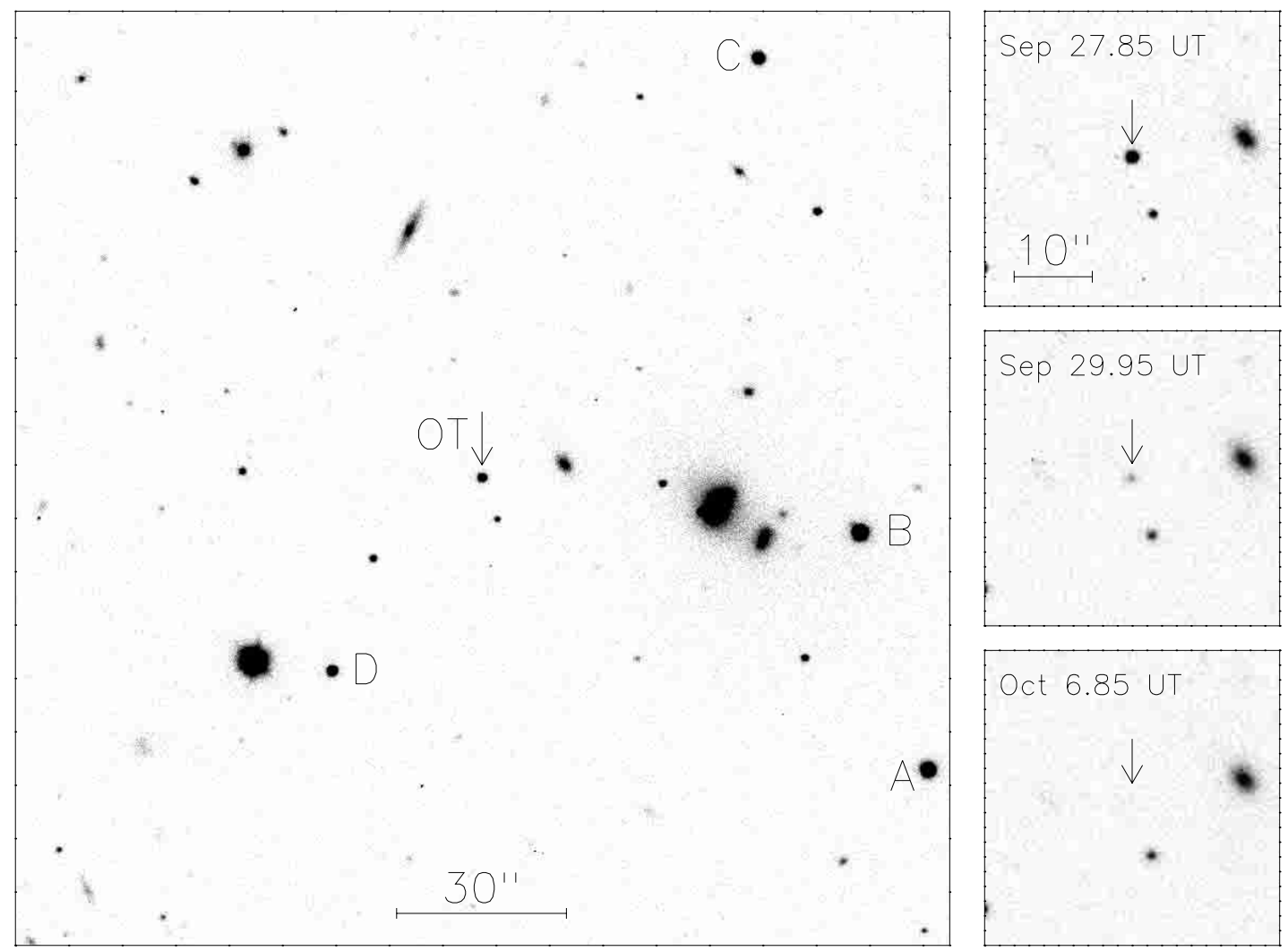

Fig. 1. Left panel: An $R$-band finding-chart for GRB 000926 centred on the position of the OT (marked with an arrow). Also marked are the four secondary calibration stars A-D used to transform the relative PSF-photometry onto the standard system. The photometric properties of the stars are given in Table 1. Right panels: Three smaller $R$-band images centred on the OT at three different epochs showing the decline of the OT.

Table 1. UBVRI magnitudes for the four secondary calibration stars A-D.

\begin{tabular}{lccccc}
\hline star/filter & $U$ & $B$ & $V$ & $R$ & $I$ \\
\hline A & $19.070 \pm 0.069$ & $18.499 \pm 0.021$ & $17.635 \pm 0.010$ & $17.025 \pm 0.021$ & $16.553 \pm 0.028$ \\
B & $19.596 \pm 0.119$ & $18.662 \pm 0.025$ & $17.508 \pm 0.010$ & $16.811 \pm 0.022$ & $16.265 \pm 0.030$ \\
C & $18.920 \pm 0.086$ & $19.047 \pm 0.036$ & $18.449 \pm 0.016$ & $18.026 \pm 0.028$ & $17.655 \pm 0.038$ \\
D & - & $20.822 \pm 0.141$ & $19.459 \pm 0.045$ & $18.529 \pm 0.065$ & $17.694 \pm 0.069$ \\
\hline
\end{tabular}

Spread Function (PSF) photometry there will be a contribution from the underlying host galaxy. The relative strength of this contribution depends on the seeing and is therefore a source of systematic errors if not corrected for. We therefore subtracted an aligned and scaled image of the host galaxy from each of the individual $R$-band images from the NOT listed in Table 2. This image of the host galaxy was obtained about 30 days after the burst when the magnitude of the OT, based on an extrapolation of the light-curve, as well as a possible underlying supernova would be negligible (see Sect. 6). We then measured the magnitude of the OT using DAOPHOT-II (Stetson 1987, 1997). The OT was last detected in the image obtained on October 2. The photometry was transformed to the standard system using photometric observations of the four secondary reference stars A-D marked in Fig. 1 obtained at the U.S. Naval Observatory Flagstaff Station.
The calibrated $U B V R I$ magnitudes of the stars $\mathrm{A}-\mathrm{D}$ are given in Table 1.

We first fitted a broken power-law

$f_{\nu}(t)=\left\{\begin{array}{l}f_{\nu}\left(t_{\mathrm{b}}\right)\left(\frac{t}{t_{\mathrm{b}}}\right)^{-\alpha_{1}}, \text { if } t \leq t_{\mathrm{b}} \\ f_{\nu}\left(t_{\mathrm{b}}\right)\left(\frac{t}{t_{\mathrm{b}}}\right)^{-\alpha_{2}}, \text { if } t \geq t_{\mathrm{b}}\end{array}\right.$

to the data, which provided a very good fit. The parameters of the fit are given in Table 3. We then followed Beuermann et al. (1999) and fitted an empirical function of the form

$f_{\nu}(t)=\left(f_{1}(t)^{-n}+f_{2}(t)^{-n}\right)^{-1 / n}$

where $f_{i}(t)=k_{i} t^{-\alpha_{i}}$ and $t$ is the time since the GRB measured in days. For large values of $n$ this function approaches the broken power-law. We performed this fit both 
Table 2. The journal of NOT, CA, INT and USNOFS observations and the results of the photometry.

\begin{tabular}{|c|c|c|c|c|}
\hline$\overline{\mathrm{UT}}$ & Obs. & mag & $\begin{array}{l}\text { Seeing } \\
(\text { arcsec) }\end{array}$ & $\begin{array}{c}\text { Exposure time } \\
(\mathrm{sec})\end{array}$ \\
\hline \multicolumn{5}{|l|}{$\overline{U-b a n d^{a}}$} \\
\hline Sep. 27.9484 & NOT & $20.437 \pm 0.047$ & 1.35 & 1000 \\
\hline Sep. 28.8291 & NOT & $21.732 \pm 0.224$ & 1.29 & 450 \\
\hline $\begin{array}{l}\text { Sep. } 29.8493 \\
\text { B-band }\end{array}$ & NOT & $22.816 \pm 0.131$ & 1.16 & 900 \\
\hline Sep. 27.8881 & NOT & $20.432 \pm 0.034$ & 1.00 & 100 \\
\hline Sep. 27.9673 & $\mathrm{CA}$ & $20.733 \pm 0.034$ & 2.42 & 1200 \\
\hline Sep. 27.9894 & $\mathrm{CA}$ & $20.727 \pm 0.038$ & 2.41 & 1200 \\
\hline Sep. 28.0057 & $\mathrm{CA}$ & $20.796 \pm 0.039$ & 2.59 & 1200 \\
\hline Sep. 29.8346 & NOT & $22.890 \pm 0.122$ & 1.19 & 500 \\
\hline Sep. 29.8688 & NOT & $22.856 \pm 0.082$ & 1.20 & 600 \\
\hline \multicolumn{4}{|l|}{$V-$ band $^{a, b}$} & 1200 \\
\hline Sep. 27.906 & INT & $19.906 \pm 0.0135$ & 1.0 & 300 \\
\hline Sep. 27.910 & INT & $19.933 \pm 0.0135$ & 1.0 & 300 \\
\hline Sep. 27.915 & INT & $19.936 \pm 0.0141$ & 1.0 & 300 \\
\hline Sep. 27.9237 & $\mathrm{CA}$ & $19.910 \pm 0.025$ & 2.22 & 900 \\
\hline Sep. 29.9557 & NOT & $22.324 \pm 0.121$ & 1.29 & 600 \\
\hline Sep. 30.839 & INT & $22.987 \pm 0.096$ & 1.0 & 600 \\
\hline \multicolumn{5}{|l|}{$R-$ band $^{a, b}$} \\
\hline Sep. 27.8547 & NOT & $19.326 \pm 0.015$ & 0.83 & 300 \\
\hline Sep. 27.8594 & NOT & $19.343 \pm 0.011$ & 0.84 & 300 \\
\hline Sep. 27.8639 & NOT & $19.322 \pm 0.011$ & 0.86 & 300 \\
\hline Sep. 27.8651 & $\mathrm{CA}$ & $19.329 \pm 0.034$ & 1.44 & 900 \\
\hline Sep. 27.8684 & NOT & $19.342 \pm 0.008$ & 0.82 & 300 \\
\hline Sep. 27.8729 & NOT & $19.349 \pm 0.011$ & 0.87 & 300 \\
\hline Sep. 27.8774 & NOT & $19.366 \pm 0.010$ & 0.90 & 300 \\
\hline Sep. 27.8820 & NOT & $19.349 \pm 0.010$ & 0.91 & 300 \\
\hline Sep. 27.892 & INT & $19.394 \pm 0.022$ & 1.0 & 300 \\
\hline Sep. 27.896 & INT & $19.411 \pm 0.022$ & 1.0 & 300 \\
\hline Sep. 27.901 & INT & $19.421 \pm 0.023$ & 1.0 & 300 \\
\hline Sep. 27.9109 & $\mathrm{CA}$ & $19.452 \pm 0.020$ & 2.01 & 900 \\
\hline Sep. 27.9658 & NOT & $19.538 \pm 0.011$ & 1.06 & 300 \\
\hline Sep. 27.9705 & NOT & $19.526 \pm 0.014$ & 1.09 & 300 \\
\hline Sep. 27.9750 & NOT & $19.567 \pm 0.015$ & 1.01 & 300 \\
\hline Sep. 27.9795 & NOT & $19.542 \pm 0.017$ & 1.02 & 300 \\
\hline Sep. 27.9840 & NOT & $19.553 \pm 0.023$ & 1.00 & 300 \\
\hline Sep. 28.097 & USNOFS & $19.71 \pm 0.03$ & 2.0 & $3 \times 600$ \\
\hline Sep. 28.8356 & NOT & $20.723 \pm 0.025$ & 1.06 & 300 \\
\hline Sep. 28.8911 & NOT & $20.774 \pm 0.036$ & 1.05 & 500 \\
\hline Sep. 28.9595 & NOT & $20.875 \pm 0.036$ & 1.19 & 600 \\
\hline Sep. 29.110 & USNOFS & $20.94 \pm 0.04$ & 1.8 & $3 \times 600$ \\
\hline Sep. 29.138 & USNOFS & $20.95 \pm 0.04$ & 1.9 & $4 \times 600$ \\
\hline Sep. 29.170 & USNOFS & $21.08 \pm 0.05$ & 2.0 & $4 \times 600$ \\
\hline Sep. 29.8414 & NOT & $21.740 \pm 0.043$ & 0.86 & 600 \\
\hline Sep. 29.9478 & NOT & $21.897 \pm 0.083$ & 1.17 & 600 \\
\hline Sep. 30.83 & NOT & $22.494 \pm 0.070$ & 0.90 & 2400 \\
\hline Oct. 2.84 & NOT & $23.514 \pm 0.153$ & 1.14 & 1500 \\
\hline Oct. 6.85 & NOT & - & 1.29 & 2700 \\
\hline Oct. 27.84 & NOT & - & 1.10 & 4700 \\
\hline Nov. 3.84 & NOT & - & 1.26 & 2400 \\
\hline Nov. 4.84 & NOT & - & 1.12 & 3600 \\
\hline \multicolumn{5}{|l|}{$I-b a n d^{a, b}$} \\
\hline Sep. 27.919 & INT & $18.959 \pm 0.029$ & 1.0 & 300 \\
\hline Sep. 27.924 & INT & $18.992 \pm 0.029$ & 1.0 & 300 \\
\hline Sep. 27.929 & INT & $19.007 \pm 0.029$ & 1.0 & 300 \\
\hline Sep. 27.9370 & CA & $18.861 \pm 0.032$ & 2.22 & 900 \\
\hline Sep. 27.9433 & NOT & $19.014 \pm 0.014$ & 0.72 & 600 \\
\hline Sep. 27.9510 & $\mathrm{CA}$ & $18.890 \pm 0.022$ & 2.16 & 900 \\
\hline Sep. 29.8608 & NOT & $21.218 \pm 0.051$ & 0.79 & 600 \\
\hline Sep. 30.839 & INT & $21.956 \pm 0.029$ & 1.0 & 600 \\
\hline
\end{tabular}

${ }^{a}$ Only for the NOT $R$-band measurements have we subtracted a galaxy image before doing the PSF photometry.

$b$ The following filters were used: NOT - all Bessel; CA - all Johnson; INT: Sloan i, Harris R \& V; USNOFS : Bessel R.

keeping $n$ fixed at 1 similar to Stanek et al. (1999) and with $n$ as a free parameter. The results of these fits are also given in Table 3 .

The $\chi^{2}$ per degree of freedom is smallest for the broken power-law fit. The data favor a very large value of $n$ as the $\chi^{2}$ is monotonically decreasing as $n$ is increased even

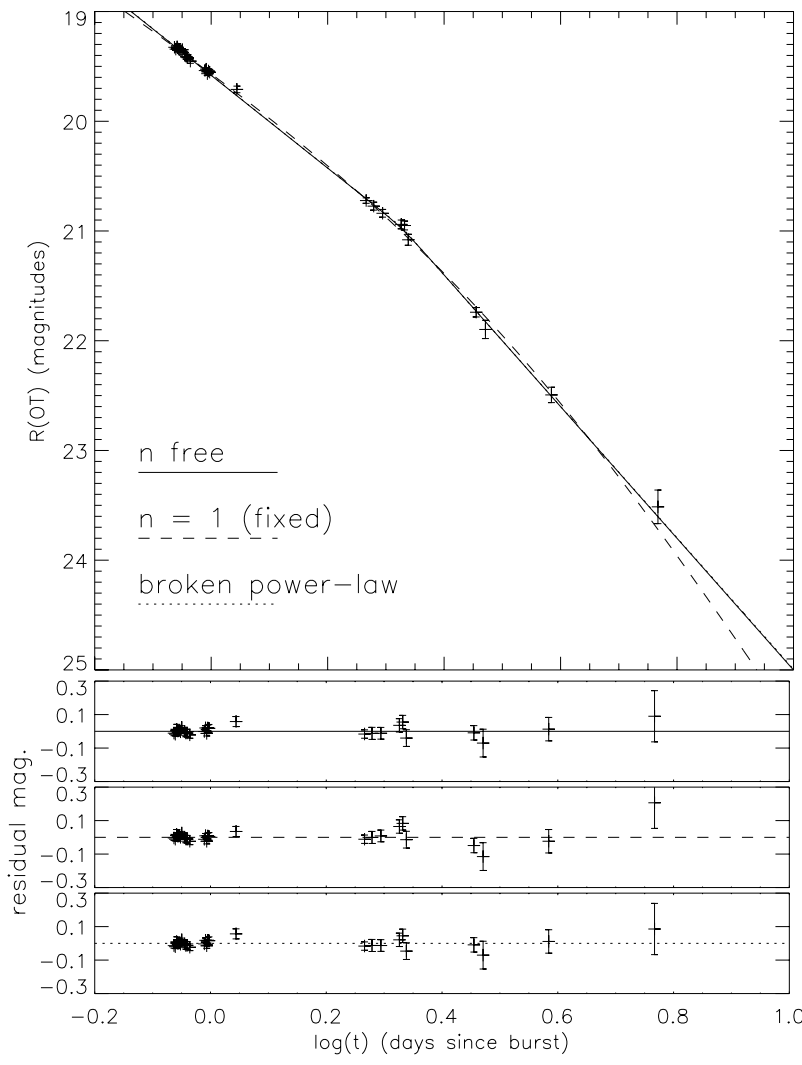

Fig. 2. The $R$-band light-curve of the OT. The upper panel show the $R$-band measurements together with the three lightcurve fits. The three lower panels shows the deviations around the fits for, from top to bottom, the broken power-law, the $n$ fixed and the $n$ free fits.

Table 3. Results of fits to the $R$-band light-curve. There were 24 degrees of freedom in the fits.

\begin{tabular}{lcccc}
\hline Fit & $\alpha_{1}$ & $\alpha_{2}$ & other & $\chi^{2} /$ d.o.f. \\
\hline$b . p .-l$. & $1.69 \pm 0.02$ & $2.39 \pm 0.09$ & $t_{\mathrm{b}}=2.12 \pm 0.09$ & 1.000 \\
$n=1$ & $1.40 \pm 0.13$ & $3.36 \pm 0.41$ & $n=1$ (fixed) & 1.263 \\
$n$ free & $1.69 \pm 0.02$ & $2.39 \pm 0.09$ & $n>7(2 \sigma)$ & 1.084 \\
\hline
\end{tabular}

for $n>100$. The $2 \sigma$ lower limit on $n$ is 7 in the sense that the difference between $\chi^{2}$ for a fit with $n=7$ and the broken power-law is 2 . This indicates that the break in the light-curve is very abrupt (within a few hours).

In conclusion, the data are best fit by a sharp break around $t=2.12 \pm 0.09$ days after the burst. In Fig. 2 we show the $R$-band light-curve of the OT together with the three fits and the residuals around the fits.

\subsection{Other optical bands}

The OT was also imaged in the $U, B, V$ and $I$ bands during the first four nights after the detection at the NOT and CA.

To precisely determine the broad band colours of the OT we used the $U B V$ and $I$ band observations obtained 
Table 4. Colours of the OT of GRB 000926 and GRB 000301C. The colours of the OT of GRB 000301C are from Jensen et al. (2001).

\begin{tabular}{lccc}
\hline Colour & GRB 000926 & $\chi^{2} /$ d.o.f. & GRB 000301C \\
\hline$U-R$ & $0.96 \pm 0.04$ & 0.59 & $0.40 \pm 0.05$ \\
$B-R$ & $1.06 \pm 0.03$ & 0.53 & $0.91 \pm 0.03$ \\
$V-R$ & $0.50 \pm 0.01$ & 0.34 & $0.44 \pm 0.05$ \\
$R-I$ & $0.48 \pm 0.01$ & 0.48 & $0.49 \pm 0.04$ \\
$R-K$ & $3.35 \pm 0.07$ & 1.87 & $2.98 \pm 0.08$ \\
\hline
\end{tabular}

at NOT and INT. The CA points were excluded since Johnson $R$ and $I$ filters are significantly different from Bessel, Cousins and Harris. We determined the colours as the offset of the broken power-law fit to the $R$-band light-curve that minimized the $\chi^{2}$ of the fit. Due to the lower error bars the magnitudes obtained on Sep. 27 have the largest weight in the fits. Therefore we do not expect a large systematic uncertainty due to emission from the underlying host galaxy. In order to minimize the effect of the host galaxy only points obtained earlier than and including September 29 were used in the fits. The $1 \sigma$ errors on the colours were determined as the colours that increased the value of $\chi^{2}$ by 1 , but the true uncertainty including calibration and systematic errors is most likely somewhat larger $(\sim 5 \%)$. For all filters $U, B, V$ and $I$ the fits were consistent with the (offset) broken power-law fit, which shows that the data within the errors (few percent) are consistent with an achromatic optical afterglow. The results are given in Table 4 .

\subsection{Infrared photometry}

The afterglow was observed in the $K^{\prime}$-band on September 29, 2000 with the IRCS instrument on the 8.2-m Subaru telescope in a seeing of about 0.7 arcsec and a total integration time of $1800 \mathrm{~s}$. No standard star observations were obtained on the same night, but fortunately there is a bright, unsaturated 2MASS source in the field which we could use for calibration. We measured the magnitude of the OT and of the 2MASS source in a 2 arcsec circular aperture. The formal photometric error-bar is less than a percent, but we estimate conservatively that the uncertainty in the colour-transformation for the IRCS instrument amounts to $0.10 \mathrm{mag}$.

The afterglow was also observed in the $J, H$ and $K$ bands with the UFTI imager on UKIRT on September 30. The final frames were accumulated in 26, 24 and 9 dithered exposures of $60 \mathrm{~s}$ duration for $J, H$ and $K$ respectively resulting in a total on-source integration time of $1.56 \mathrm{ks}, 1.44 \mathrm{ks}$ and $540 \mathrm{~s}$, all in photometric conditions. Employing standard procedures these frames were reduced, combined and calibrated using observations of UKIRT faint standards bracketing the science exposures. The final frames have only a (for this instrument) modest seeing of $0.55-0.60^{\prime \prime} F W H M$ due to the relatively high
Table 5. Log of $I R$ observations.

\begin{tabular}{lllcc}
\hline UT (Sep) & filter/Obs. & mag & $\begin{array}{c}\text { Seeing } \\
\text { (arcsec) }\end{array}$ & $\begin{array}{c}\text { Exp. time } \\
\text { (sec) }\end{array}$ \\
\hline 30.276 & J/UKIRT & $20.83 \pm 0.15$ & 0.6 & 1560 \\
30.250 & H/UKIRT & $19.46 \pm 0.10$ & 0.6 & 1440 \\
29.24 & K'/SUBARU & $17.86 \pm 0.10$ & 0.7 & 1800 \\
30.301 & K/UKIRT & $18.66 \pm 0.11$ & 0.6 & 540 \\
\hline
\end{tabular}

airmass of the observations, $1.5-2$, but clearly detect the OT in all three passbands. The magnitude of the OT was again measured using aperture photometry. The results of the IR photometry is presented in Table 5 .

Using the standard star calibrated UKIRT $K$-band observations we confirmed from faint objects visible in both the UKIRT and SUBARU images that the calibration of the SUBARU images is consistent with that of the UKIRT $K$-band observations.

\section{Spectral energy distribution of the afterglow}

Also shown in Table 4 are the colours (in the same bands) of the OT of GRB 000301C, which was also discovered at the NOT and had a very similar redshift as GRB 000926 (Jensen et al. 2001; Møller et al., in prep). As seen, the OTs of GRB 000926 and GRB 000301C had very similar colours in the optical red bands, whereas in the blue bands and in $R-K$ the OT of GRB 000926 was significantly redder than that of GRB 000301C. In order to test whether this difference is intrinsic to the bursts or caused by a larger extinction along the line of sight to GRB 000926 we follow Jensen et al. (2001) and constrain the extinction by fitting different extinction laws to the SED.

To construct the SED we first used the colours given in Table 4 for the observed $U$ to $I$ bands (normalised to Sep. $27.9 \mathrm{UT}$ ). The $J, H$ and $K$-observations were obtained on Sep. 30.3 where the host galaxy possibly contributed significantly to the flux. In order to estimate the effect of the host galaxy we used the SEDs for galaxies at redshifts $z=2-3$ given by Dickinson (2000, their Fig. 2). By normalising these galaxy SEDs to the observed $R(A B)=24.04 \pm 0.15$ for the host galaxy (see Sect. 6 below) we derived magnitudes for the host galaxy which translate into estimated corrections at Sep. $30.3 \mathrm{UT}$ of $\Delta J=+0.14 \pm 0.08, \Delta H=+0.07 \pm 0.06$ and $\Delta K=$ $+0.05 \pm 0.04$. The $J H K$ magnitudes were then shifted to Sep. 27.9 UT using the broken power-law fit to the lightcurve given in Table 3 (assuming that the burst evolved achromatically). After this the $U B V R I J H K$ magnitudes were corrected for foreground extinction, using a value of $E(B-V)=0.023$ from Schlegel et al. (1998), and transformed to the $A B$ system. For the optical bands we used the transformations given by Fukugita et al. (1995): $I(A B)=I+0.43, R(A B)=R+0.17, V(A B)=V-0.02$ $B(A B)=B-0.14$, and $U(A B)=U+0.69$. We assigned uncertainties of $0.05 \mathrm{mag}$ to the $B V R$ and $I$ $A B$ magnitudes as an estimate of the uncertainty in the 
Table 6. The fits to the spectral energy distribution of GRB000926.

\begin{tabular}{lccc}
\hline & $\chi^{2} /$ d.o.f. & $\beta$ & $A_{V}$ \\
\hline No extinction & 3.20 & $1.42 \pm 0.06$ & 0 \\
\hline Pei (1992), MW & & & $<0$ \\
Pei (1992), LMC & 2.61 & $0.98 \pm 0.23$ & $0.27 \pm 0.12$ \\
Pei (1992), SMC & 1.71 & $1.00 \pm 0.18$ & $0.18 \pm 0.06$ \\
\hline
\end{tabular}

transformation. For $U$ band we assigned an uncertainty of $0.10 \mathrm{mag}$ to the $A B$ magnitude since this band is more difficult to calibrate (Bessel 1990; Fynbo et al. 1999, 2000c). For the $I R$ bands we used the transformations given in Allen (2000): $K(A B)=K+1.86, H(A B)=H+1.35$, and $J(A B)=J+0.87$. We then calculated the specific flux using $F_{\nu}=10^{-0.4 \times(A B+48.60)}$. Finally, the wavelengths corresponding to our $U B V R I J H K$ measurements were blueshifted to the GRB rest frame. As it can be seen in Fig. 3 the spectral energy distribution is clearly bending from the $U$ to the $K$-band. This bend can be naturally explained by the presence of intrinsic extinction at $z=2.037$. The $J$-point is falling significantly below the trend of all the other points. The reason for this is not understood, but we have decided not to include this point in the analysis. Including the point does not change any of the conclusions, but it increases the $\chi^{2}$ of the fits.

We next fitted the function $F_{\nu} \propto \nu^{-\beta} \times 10^{\left(-0.4 A_{\nu}\right)}$ to the SED. Here, $\beta$ is the spectral index and $A_{\nu}$ is the extinction in magnitudes at frequency $\nu$. We have considered the three extinction laws $\left(A_{\nu}\right.$ as a function of $\left.\nu\right)$ given by Pei (1992), i.e. for the Milky-Way (MW), Large Magellanic Cloud (LMC) and the Small Magellanic Cloud (SMC). In the three cases the dependence of the extinction with $\nu$ have been parameterized in terms of (restframe) $A_{V}$. Thus, our fits allow us to determine $\beta$ and $A_{V}$ simultaneously. Finally, we also considered the no-extinction case where $F_{\nu}$ was fitted by a straight line in log-log space.

The parameters of the fits are shown in Table 6 . For the no-extinction case we find a value of $\beta$ consistent with that of Price et al. (2001). As for GRB 000301C the best fit was achieved for a SMC extinction law. We derive a modest extinction of $A_{V}=0.18 \pm 0.06$ (restframe $V$ ) and a spectral index $\beta=1.00 \pm 0.18$. For GRB 000301C Jensen et al. (2001) found $\beta=0.70 \pm 0.09$. Therefore, GRB000926 was indeed intrinsically redder than GRB 000301C.

In the upper panel of Fig. 3 we show the fits using the LMC and SMC extinction laws and the no-extinction case. For the redshift of GRB 000926 (as for that of GRB 000301C) the interstellar extinction bump at $2175 \AA$ is shifted into the $R$-band. This absorption bump is very prominent for the MW, moderate for the LMC and almost nonexistent for the SMC extinction curve. Thus, for a chemically rich environment, like the MW, we should expect a prominent extinction bump at $2175 \AA$ (near the observed $R$-band). The data points in Fig. 3 show that there is no strong absorption bump near the $R$-band, which makes the fit for the MW (see Table 6) inconsistent with the data. In fact, the best MW fit implies a (unphysical) negative extinction. To illustrate the problem with the MW extinction curve we have in the lower panel of Fig. 3 plotted a $\beta=1$ power-law SED extincted by a $A_{V}=0.2 \mathrm{MW}$ extinction curve. As seen, the shape of this extinction curve is incompatible with the data. In the Milky Way the extinction curve can be different mainly for stars located in star-forming regions (Baade \& Minkowski 1937; Whittet 1992) in the sense that the shape of the bump at $2175 \AA$ is different and more importantly the curve is almost flat in the rest-frame UV at $\log (\nu)>15.1$. This is where the curvature is most pronounced in Fig. 3 and therefore such an extinction curve is also not compatible with the data (see also Price et al. 2001)

In conclusion, as in the case of GRB000301C, the SED supports a scenario of a host in an early stage of chemical enrichment.

\section{The host galaxy}

In the images obtained on October 6 the emission at the position of the OT appeared elongated although at low signal-to-noise ratio. We therefore obtained further deep imaging on October 27 and November 3 and 4. A total of 3 hours of $R$-band imaging was obtained under dark/grey sky conditions and a seeing varying between 1.0 and 1.3 arcsec. We subsequently combined these images using the code described in Møller \& Warren (1993). The $F W H M$ of point sources in the combined image was 1.18 arcsec. In the combined image an extended object directly underneath the position of the OT was clearly detected. A contour image showing this extended emission is shown as Fig. 6. Although the seeing was not optimal, the object is resolved into several compact knots covering a region of total extension of about 5 arcsec. This is more clearly visible in images of the host galaxy + OT obtained with the Hubble Space Telescope presented by Price et al. (2001). In order to determine the position of the OT relative to these compact knots we measured the position of the OT relative to point-sources in the vicinity in the first image obtained on September 27 and in the combined image. The position of the OT in the combined image could in this way be determined with a conservatively estimated uncertainty of $0.02 \operatorname{arcsec}(10 \%$ of a pixel). The position of the OT is within the errors spatially coincident with one of the knots of the extended emission. As the OT, based on an extrapolation of the light-curve, is expected to be much fainter than this knot (by several magnitudes) we conclude that at least this particular knot is related to the host galaxy of GRB 000926. An underlying supernova (SN) even if similar to SN1998bw would also be much fainter than the observed knot, especially since the $R$-band corresponds to the rest-frame UV around $2100 \AA$ where SNe are intrinsically faint. Whereas it is possible that all the compact knots are either part of the host galaxy or several galaxy sub-clumps in the process of merging, we cannot exclude that some of the emission is from objects 

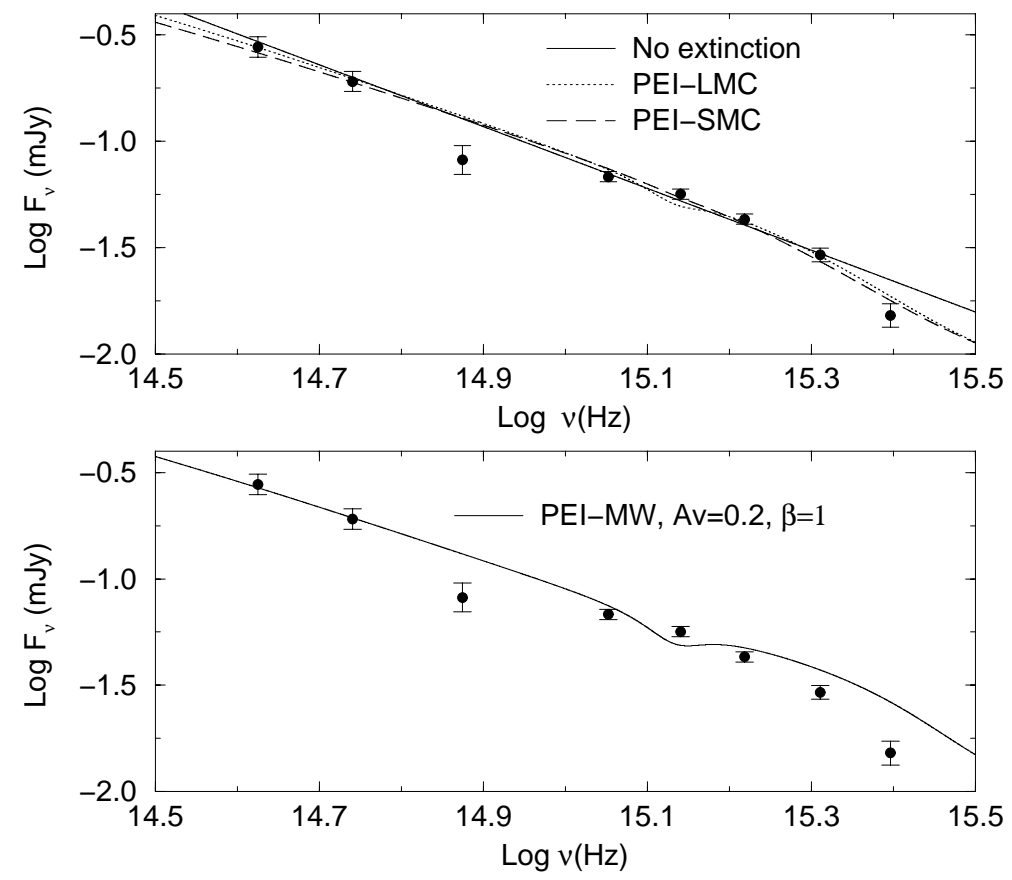

Fig. 3. The observed specific flux $F_{\nu}$ vs. the rest-frame frequency $\nu$ normalised to Sep. 27.9 UT. The spectral energy distribution of the afterglow from $K$ to $U$ is clearly curved. In the upper panel we show that this curvature can be well fitted by an underlying $\beta=1$ power-law SED with a SMC or LMC extinction law with $A_{V}=0.2$. In the lower panel we show that the MW extinction law is inconsistent with the data.

lying at other distances along the line of sight (although there are no intervening absorption systems in the spectrum of the OT, Møller et al., in prep). If all the emission is from the host galaxy of GRB 000926 then the extension of the galaxy would be similar to the Ly- $\alpha$ emitting region of the galaxy $\mathrm{S} 4$ at $z=1.93$ towards Q0151+048A (Fynbo et al. 1999).

The total magnitude of the extended emission in a circular aperture with diameter 4.7 arcsec is $R=23.87 \pm 0.15$. Assuming that all this emission comes from the host galaxy we can get an estimate of the star-formation-rate (SFR) of the galaxy. The restframe UV continuum in the range $1500 \AA-2800 \AA$ can be used as a SFR estimator if one assume that the star-formation is continuous over a time scale of more than $10^{8}$ years. Kennicutt (1998) provides the relation

$\operatorname{SFR}\left(M_{\odot} \mathrm{yr}^{-1}\right)=1.4 \times 10^{-28} \times L_{\nu}$,

where $L_{\nu}$ is the luminosity in the $1500 \AA-2800 \AA$ range measured in erg s ${ }^{-1} \mathrm{~Hz}^{-1}$. The observed $R$-band corresponds to the rest-frame UV continuum around $2100 \AA$, which falls well within this range. To derive $L_{\nu}$ we first converted our $R$-band magnitude to $R(A B)$ using $R(A B)=R+0.17$ (Fukugita et al. 1995). Then we used the definition of the $A B$ magnitude to derive the observed flux $\left(F_{\nu}=10^{-0.4 \times(R(A B)+48.6)}\right)$ and finally the luminosity distance in our assumed cosmology $\left(d_{\text {lum }}=5.28 \times 10^{28} \mathrm{~cm}\right)$ to derive $L_{\nu}$ :

$$
\begin{aligned}
L_{\nu} & =F_{\nu} \times 4 \pi d_{\text {lum }}^{2} /(1+z) \\
& =(1.02 \pm 0.15) \times 10^{29} \mathrm{erg} \mathrm{s}^{-1} \mathrm{~Hz}^{-1},
\end{aligned}
$$

where the factor $(1+z)^{-1}$ corrects for the fact that $L_{\nu}$ is a specific luminosity (not a bolometric luminosity). Using the relation of Kennicutt (1998) we find a SFR of $14 M_{\odot} \mathrm{yr}^{-1}$. If the extinction derived in Sect. 5 is valid for the galaxy as a whole then we estimate an extinction in the observed $R$-band of $0.56 \pm 0.20 \mathrm{mag}$, which means that the SFR should be increased by a factor of $\sim 1.7$. This SFR is a high compared to that of the Milky Way, but it falls in the low end of the range of SFRs of Lyman-Break galaxies (Pettini et al. 1998).

\section{Discussion}

\subsection{Interpretation of the light-curve}

The temporal behavior of the optical afterglow of GRB 000926 is a clear and unambiguous example of a broken power-law decay. The fits described in Sect. 4.1 show that the break occurred abruptly. The late time decay slope of $\alpha_{2}=2.39 \pm 0.09$ is very similar to the late time decay slopes of other well studied broken or fast decaying light-curves (see e.g. Andersen et al. 2000, their Fig. 4 and Table 4). This striking uniformity suggest a common physical scenario for the late stage of the decays, which most likely is a common value of the index of the electron energy distribution (see also Sari et al. 1999; Freedman \& Waxman 2001).

The increase $\Delta \alpha=\alpha_{2}-\alpha_{1}$ from the early to the late time decay slope is different for different physical models for GRB afterglows. For GRB 000926 we find $\Delta \alpha=0.70 \pm 0.09$ from the broken power-law fit. This measurement we compare with different models predicting 


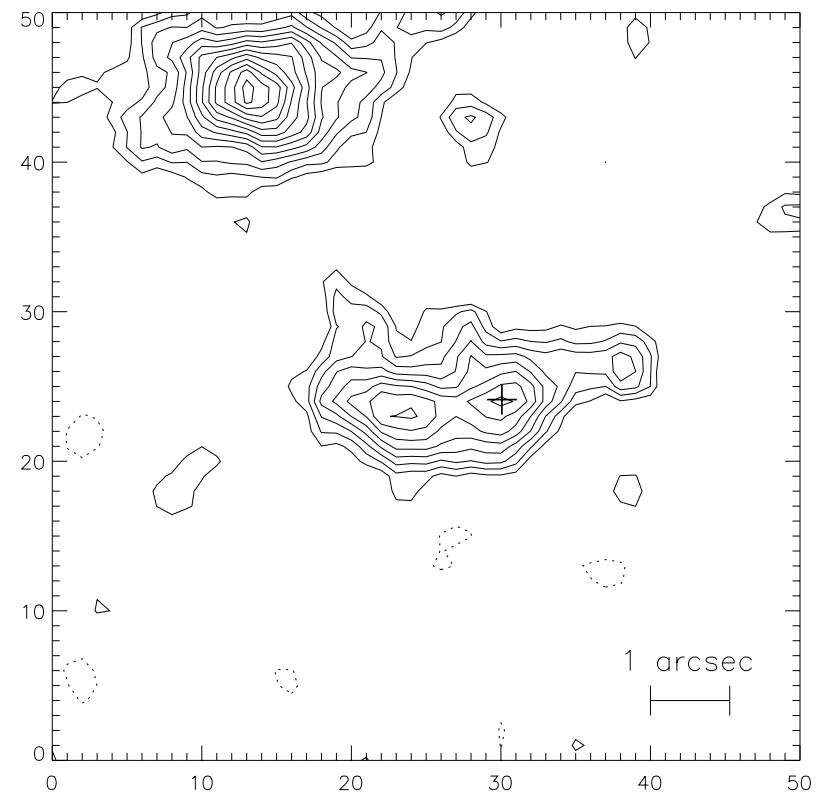

Fig. 4. A contour plot of a $9.5 \times 9.5 \operatorname{arcsec}^{2}$ region centred on the probable host galaxy of GRB 000926. The image has been smoothed with a Gaussian filter with a width of 2 pixels $(0.39$ arcsec $)$. The contour levels are $-2,2,3.5,5,6.5, \ldots \times$ $1 \sigma$ of the sky-noise, with the dotted contours being negative. East is left and north is up. The cross marks the position of the OT. The error on the position of the OT is 0.10 pixels corresponding to 0.02 arcsec.

broken light-curves: i) If the frequency separating fast cooling and slow cooling electrons moves through the optical part of the electromagnetic spectrum at $t_{\mathrm{b}}$, the resulting light curve would steepen by $\Delta \alpha \sim 0.25$ (Sari et al. 1998); ii) If a spherical fireball slows down to a non-relativistic expansion (Dai \& Lu 1999) then $\Delta \alpha=$ $\left(\alpha_{1}+3 / 5\right)=1.09$ for our value of $\alpha_{1}$; iii) If the outflow is collimated with a fixed opening angle, the break in the light curve occurs when the relativistic beaming of the synchrotron radiation becomes wider than the jet opening angle with a predicted steepening of $\Delta \alpha=3 / 4$ (Mészáros \& Rees 1999); iv) finally, if the afterglow arises in a sideways expanding jet, the steepening will be $\Delta \alpha=$ $\left(1-\alpha_{1} / 3\right)=0.44$ (Rhoads 1999$)$ for our value of $\alpha_{1}$. The above estimates all assume a constant mean density distribution of the ambient medium. Only model iii), i.e. a jet with fixed opening angle, is consistent with the observed value of $\Delta \alpha=0.70 \pm 0.09$. This model predicts a spectral slope of the afterglow of $\beta=2 \alpha_{1} / 3=1.13 \pm 0.01$, which is consistent with the $\beta=1.00 \pm 0.18$ from the multi-band photometry. If the density of the surrounding medium was that of stellar wind $\left(n \propto r^{-\delta}\right.$ with $\left.\delta=2\right)$ we expect $\Delta \alpha=\frac{3-\delta}{4-\delta}=0.50$ (Mészáros \& Rees 1999; Jaunsen et al. 2001), which is excluded by the data at the $2.2 \sigma$ level.

\subsection{Comparison with GRB $000301 C$}

Even though the gamma-ray emission of GRB 000301C (Jensen et al. 2001) was of about 10 times shorter duration than that of GRB 000926, the fact that they have nearly identical redshifts of $z=2.0375 \pm 0.0007$ for GRB 000926 (Møller et al., in prep) and $z=2.0404 \pm 0.0008$ for GRB 000301C (Jensen et al. 2001) makes it very convenient to compare the two. Both GRB 000301C and GRB 000926 displayed broken power-law decays. For the OT of GRB 000301C Jensen et al. (2001) determined $\beta=0.70 \pm 0.09$ and $\Delta \alpha=1.57 \pm 0.18$. In this case the best model is that of a side-ways expanding jet in a medium of constant density, whereas a jet with fixed opening angle is not consistent with the data. Therefore, even though the two bursts appear similar they cannot be explained by the same model.

GRB 000301C and GRB 000926 have very different host galaxies. The host galaxy of GRB 000301C remains undetected despite a very deep detection limit of $R=28.5$ (Fruchter et al. 2000a; Smette et al. 2001), whereas the host galaxy of GRB 000926 is relatively bright at $R=23.87 \pm 0.15$ (Sect. 6). Hence, the host galaxy of GRB 000926 is more than 70 times brighter than that of GRB 000301C. In the same way GRB 990123 and GRB 990510 occured at nearly identical redshifts $(z \approx 1.6)$ and the host galaxy of the former is more than 30 times brighter than the latter (Holland \& Hjorth 1999; Fruchter et al. 1999, 2000b). If GRBs indeed trace star-formation these observations indicate that at these redshifts galaxies covering a broad range of luminosities contribute significantly to the over-all density of star formation. Furthermore, as the observed $R$-band flux is proportional to the star formation rate, there must be $1-2$ orders of magnitude more galaxies at the $R=28$ level than at the $R=24$ level at $z \approx 2$. Otherwise it would be unlikely to detect $R=28$ galaxies as GRB hosts (under the assumption that GRBs trace star-formation). An alternative explanation is that the faint host galaxies of GRB 000301C and GRB 990510 are faint at restframe UV wavelengths due to massive extinction similar to some sources selected in the sub-mm range (e.g. Ivison et al. 2000). However, the low extinction derived from the optical properties of the GRB 000301C afterglow argues against this explanation at least for this particular burst.

Acknowledgements. Most of the optical data presented here have been taken using ALFOSC, which is owned by the Instituto de Astrofisica de Andalucia (IAA) and operated at the Nordic Optical Telescope under agreement between IAA and the NBIfAFG of the Astronomical Observatory of Copenhagen. UKIRT is operated by the Joint Astronomy Centre on behalf of the Particle Physics and Astronomy Research Council of the United Kingdom. JUF and THD acknowledges enthusiastic help and support from C. Møller and I. Svärdh during the hectic moments of finding the OT by comparison with DSS-plates. JUF acknowledges $\mathrm{H}$. O. Fynbo for introducing him to CERNs MINUIT fitting programme. JG acknowledges the receipt of a Marie Curie Research Grant from the European Commission. MIA acknowledges the Astrophysics group of the Physics dept. of University of Oulu for support of his work. IRS acknowledges support from a Royal Society URF. IB was supported by Pôle d'Attraction Interuniversitaire, P4/05 (SSTC, Belgium). 
JMCC acknowledges the receipt of a FPI doctoral fellowship from Spain's Ministerio de Ciencia y Tecnología. KH is grateful for Ulysses support under JPL Contract 958056, and for NEAR support under NASA grants NAG5-9503 and NAG53500. Additionally, the availability of the GRB Coordinates Network (GCN) and BACODINE services, maintained by Scott Barthelmy, is greatly acknowledged. We acknowledge the availability of POSS-II exposures, used in this work; the Second Palomar Observatory Sky Survey (POSS-II) was made by the California Institute of Technology with funds from the National Science Foundation, the National Aeronautics and Space Administration, the National Geographic Society, the Sloan Foundation, the Samuel Oschin Foundation, and the Eastman Kodak Corporation. We acknowledge the availability of the 2MASS catalogs. This work was supported by the Danish Natural Science Research Council (SNF).

\section{References}

Allen, C. W. 2000, Allen's Astrophysical Quantities, 4th edition 2000, ed. A. N. Cox

Adelberger, K., \& Steidel, C. C. 2000, ApJ, 544, 218

Andersen, M. I., Hjorth, J., Pedersen, H., et al. 2000, A\&A, $364, \mathrm{~L} 54$

Baade, W., \& Minkowski, R. 1937, ApJ, 86, 123

Beuermann, K., Hassman, F. V., Reinsch, K., et al. 1999, A\&A, $352, \mathrm{~L} 26$

Bessel, M. S. 1990, PASP, 281, 817

Bloom, J. S., Kulkarni, S. R., \& Djorgovski, S. G. 2000, AJ, in press [astro-ph/0010176]

Castro-Tirado, A. J. 2001, to appear in the ESA-SP Proc. of the INTEGRAL symposium, in press [astro-ph/0102122]

Costa, E., Frontera, F., Heise, J., et al. 1997, Nature, 387, 783

Dai, C. G., \& Lu, T. 1999, ApJ, 519, L155

Dall, T., Fynbo, J. U., \& Pedersen, H., et al. 2000, GCN, 804

Dickinson, M. 2000, PTRSL, 358, 2001

Djorgovski, S. G., Pahre, M. A., Bechtold, J., \& Elston, R. 1996, Nature, 382, 234

Deutsch, E. W. 1999, AJ, 118, 1882

Ellison, S. L., Pettini, M., Steidel, C. C., \& Shapley, A. E. 2001, ApJ, 549, 770

Fynbo, J. U., Møller, P., \& Warren, S. J. 1999, MNRAS, 305, 849

Fynbo, J. U., Thomsen, B., \& Møller, P. 2000a, A\&A, 353, 457

Fynbo, J. U., Møller, P., Gorosabel, J., et al. 2000b, GCN, 825

Fynbo, J. U., Burud, I., \& Møller, P. 2000c, A\&A, 358, 88

Frail, D., et al. 2000, GCN, 805

Freedman, D. L., \& Waxman, E. 2001, ApJ, 547, 922

Fruchter, A., Thorsett, S. E., Metzger, M. R., et al. 1999, ApJ, 519, L13

Fruchter, A., Metzger, M., \& Petro, L. 2000a, GCN, 701

Fruchter, A., Hook, R., \& Pian, E. 2000b, GCN, 757

Fukugita, M., Shimasaku, K., \& Ichikawa, T. 1995, PASP, 107, 945

Gorosabel, J., Castro Ceŕon, J. M., Castro-Tirado, A. J., et al. 2000, GCN, 803

Hjorth, J., Jensen, B. L., Pedersen, H., et al. 2000, GCN, 809

Holland, S., \& Hjorth, J. 1999, A\&A, 344, L67
Hu, E. M., Cowie, L. L., \& McMahon, R. G. 1998, ApJ, 502, L99

Hughes, D. H., Serjeant, S., Dunlop, J., et al. 1998, Nature, 394,241

Hurley, K. 2000, GCN, 801 and 802

Ivison, R. J., Smail, I., Barger, A. J., et al. 2000, MNRAS, 315, 209

Jaunsen, A. O., Hjorth, J., Björnson, G., et al. 2001, ApJ, 546, 127

Jensen, B. L., Fynbo, J. U., Gorosabel, J., et al. 2001, A\&A, 369,373

Kennicutt, R. C. 1998, ARA\&A, 36, 189

Kudritzki, R.-P., Méndez, R. H., Feldmeier, J. J., et al. 2000, ApJ, 536, 19

Kulkarni, S. R., Berger, E., Bloom, J. S., et al. 2000, To appear in Proc. of the 5th Huntsville GRB Symposium [astro-ph/0002168]

Lamb, D. Q., \& Reichart, D. E. 2000, ApJ, 536, L1

Metzger, M. R., Djorgovski, S. G., Kulkarni, S. R., et al. 1997, Nature, 387,878

Mészáros, P., \& Rees, M. J. 1999, MNRAS, 306, 3, L39

Møller, P., \& Warren, S. J. 1993, A\&A, 270, 43

Møller, P., \& Warren, S. J. 1998, MNRAS, 299, 661

van Paradijs, J., Groot, P. J., Galama, T., et al. 1997, Nature, 386,686

Peacock, J. A., Rowan-Robinson, M., Balin, A., et al. 2000, MNRAS, 318, 535

Pei, Y. C. 1992, ApJ, 395, L30

Pettini, M., Kellogg, M., Steidel, C. C., et al. 1998, ApJ, 508, 539

Piro, et al. 2000, GCN, 812, 832

Price, P. A., Harrison, F. A., Galama, T. J., et al. 2001, ApJL in press [astro-ph/0012303]

Rhoads, J. E. 1999, ApJ, 525, 737

Sari, R., Piran, T., \& Narayan, R. 1998, ApJ, 497, L17

Sari, R., Piran, T., \& Halpern, J. P. 1999, ApJ, 519, L17

Schlegel, D. J., Finkbeiner, D. P., \& Davis, M. 1998, ApJ, 500, 525

Smail, I., Ivison, R. J., \& Blain, A. W. 1997, ApJ, 490, L5

Smette, A., Fruchter, A. S., Gull, T. R., et al. 2001, ApJ in press [astro-ph/0007202]

Stanek, K. Z., Garnavich, P. M., Kaluzny, J., Pych, W., \& Thompson, I. 1999, ApJ, 522, L39

Steidel, C. C., Giavalisco, M., Pettini, M., Dickinson, M., \& Adelberger, K. 1996, ApJ, 462, L17

Steidel, C. C., Adelberger, K., Shapley, A. E., et al. 2000, ApJ, 532,170

Stetson, P. 1987, PASP, 99, 191S

Stetson, P. 1997, User's Manual for DAOPHOT II

van der Werf, P. P., Knudsen, K. K., Labbé, I., \& Franx, M. 2000 , in The far-infrared and submillimeter spectral energy distributions of active and starburst galaxies, ed. I. van Bemmel, B. Wilkes, \& P. Barthel, New Astron. Rev., in press

Whittet, D. C. B. 1992, Dust in the Galactic Environment, (IOP Publishing Ltd), 73

Wolfe, A. M., Lanzetta, K. M., Foltz, C. B., \& Chaffee, F. H. 1995, ApJ, 454, 698 